\title{
Silicone granuloma mimicking lymphatic metastases in a patient with breast cancer
}

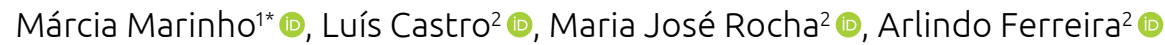

\section{ABSTRACT}

Silicone breast implants are commonly used, even for reconstruction after mastectomy in malignant disease. In this setting, the presence of suspicious lymphadenopathy should be investigated, because it could represent disease progression. A case of a woman with left breast cancer (more than 20 years ago) and prosthesic reconstruction is reported. She developed a second breast cancer on the opposite side. During follow up, a suspicious lymphadenopathy was seen in the computed tomography scan, but the final diagnosis corresponded to a siliconoma. Silicone granuloma is a difficult diagnosis in these cases, but must be considered.

KEYWORDS: breast implants; adverse effects; breast neoplasm; surgery; granuloma; diagnostic imaging; woman.

\section{INTRODUCTION}

Silicone breast implants are commonly used for breast augmentation and also in reconstruction procedures, including those after mastectomy for oncologic purposes ${ }^{1}$.

Leakage from either ruptured or intact implants can occur, stimulating granulomatous foreign body reaction. The resulting silicone granuloma, also known as siliconoma, corresponds to the inflammatory response to the free liquid silicone but could be misinterpreted as a malignant situation ${ }^{2-4}$.

Siliconomas can occur locally (manifesting as lymphadenopathy) or present at distant sites (rare cases in lower limbs and vulva have been already described) because the silicone polymer is a lipid soluble and therefore its migration in fatty tissue can easily take place ${ }^{5,6}$.

In patients with breast cancer submitted to reconstruction with silicone implants after mastectomy, the presence of siliconomas could mimic a progression of the disease. Careful evaluation is needed and the differential diagnosis must take into consideration this benign pathology.

\section{CASE REPORT}

A 66-year-old female patient with a previous left mastectomy in 1995 for neuroendocrine carcinoma (T2N0M0) was now referred to our institution for abnormal mammography of the right breast.
The neuroendocrine carcinoma was treated with chemotherapy and hormone therapy with tamoxifen. A breast reconstruction with silicone implant on the left side and a symmetrizing surgery on the right breast were performed.

In 2012, corrective surgery was done due to fibrous encapsulation of the implant.

In February 2018, the patient was referred for polymorphic microcalcifications in the upper external periareolar region of the right breast causing a dystrophic aspect on the mammogram. These alterations were not present in the previous exams.

On clinical examination, no alterations in inspection nor solid mass were palpable in both breasts. The ultrasound showed no abnormalities.

A stereotactic biopsy was performed and the histological exam revealed ductal carcinoma in situ (DCIS), nuclear grade 2 with $>90 \%$ of estrogen receptors positivity. A tumorectomy was conducted with the neoplasia adjacent to the lower surgical margin and one millimeter $(\mathrm{mm})$ apart from the medial one. The microcalcifications were present in the histological exam.

The case, pTis (DCIS) Nx, was discussed by a multidisciplinary team and it was decided to proceed with radiotherapy (RT) and hormone therapy.

In the planning computed tomography (CT) scan prior to the RT session, a suspicious lymphadenopathy of the internal mammary

${ }^{1}$ Centro Hospitalar de Vila Nova de Gaia/Espinho - Porto, Portugal.

${ }^{2}$ Hospital de Braga - Braga, Portugal

*Corresponding author: marciasilvamarinho@gmail.com

Conflict of interests: nothing to declare.

Received on: 03/14/2020. Accepted on: 06/11/2020. 
lymph nodes was identified (Figure 1). To confirm the findings, a CT scan with contrast was performed and showed an apparent intact silicone implant, as well as lymph nodes in both internal mammary chains (Figure 2), with $15 \mathrm{~mm}$ maximum diameter on the left side.

A core needle biopsy was performed (Figure 3) and the histological exam revealed "vacuolated histiocytes with little birefringent

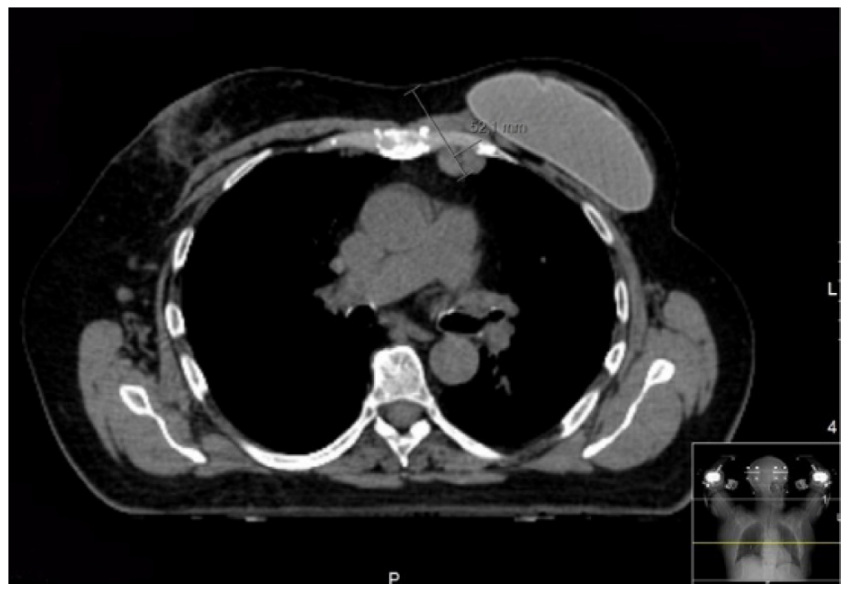

Figure 1. Planning computed tomography scan prior to radiotherapy (coronal plan): lymphadenopathy of the internal mammary lymph nodes on the left side.

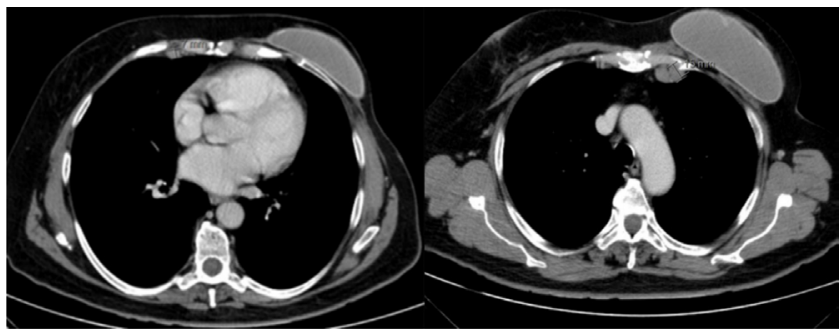

Figure 2. Contrast computed tomography scan (coronal plan): lymph nodes in both internal mammary chains, the biggest one on the left side with $15 \mathrm{~mm}$.

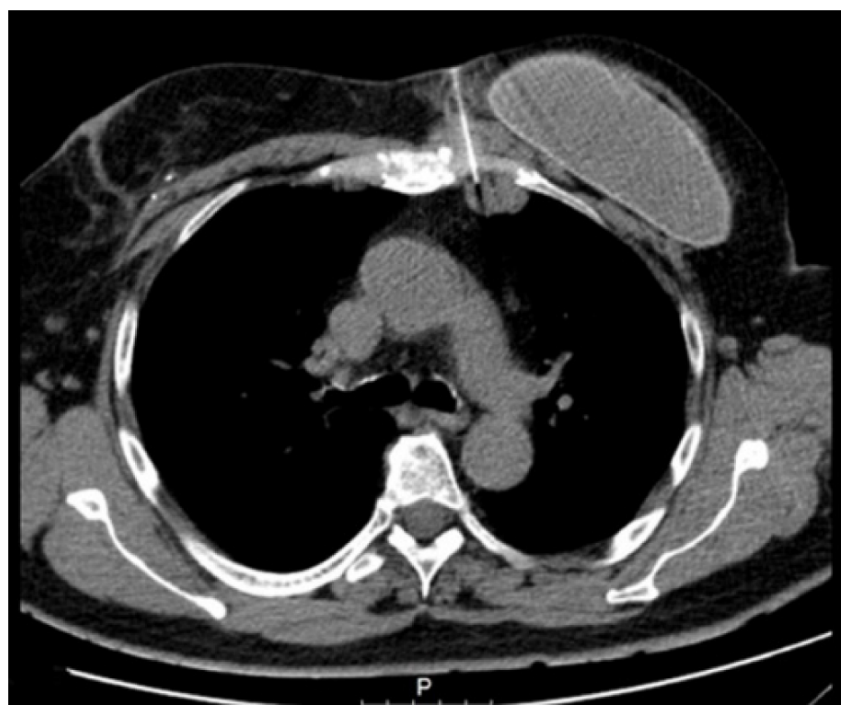

Figure 3. Core needle biopsy of the suspicious lymphadenopathy. material in polarized light and multinucleated giant cells with vacuoles of different sizes and asteroid bodies; compatible with silicone granuloma".

The patient underwent successful RT treatment. Currently, under hormone therapy, the patient is being followed up (two years) without complications.

The presence of suspicious lymph nodes in a breast cancer case could change the staging and consequently, the strategic therapy. In a patient with silicone breast implants, silicon granulomas must be considered in the differential diagnosis of suspicious lymphadenopathy.

\section{DISCUSSION}

Silicone granulomas are benign lesions that could have a similar presentation to malignancy.

In patients with breast cancer and silicone implants, the presence of lymphadenopathy might not always correspond to a progression of the disease, but instead to a siliconoma. Therefore, clinicians must be aware of this condition and consider it in the differential diagnosis ${ }^{3,6}$.

Silicone material could migrate even without clear evidence of implant rupture. The migration mechanism is still unknown, but it has been suggested that absorbed silicone molecules may follow vascular spread or travel with lymphatic flow ${ }^{5}$.

Magnetic resonance imaging (MRI) findings could include evidence of implant collapse and also free silicone particles outside the prosthetic shell ${ }^{7}$. Sonographic evaluation may reveal echogenic lesions with a "snowstorm" appearance, but there are no specific findings. Positron emission/ computed tomography (PET CT) in patients with siliconomas may be falsely positive?

Pathological tissue specimens remain the gold standard for diagnosis of siliconomas. Histological findings include foamy macrophages and refractile droplets of clear material?.

In conclusion, silicone granulomas are benign lesions rarely reported in the literature, which could nonetheless occur in patients with silicone implants, either for breast augmentation or reconstruction in oncologic patients. These lesions could be easily misinterpreted as a malignancy progression in breast cancer patients with silicone implants. Although this pathology demands a high grade of suspicion, clinicians should consider it in the differential diagnosis for proper staging and treatment of oncologic patients.

\section{AUTHORS' CONTRIBUTIONS}

M.M.: conceptualization, data curation, formal analysis, investigation, methodology, writing - original draft, writing - review \& editing.

L.C.: data curation, visualization, validation, review \& editing. M.J.R.: visualization, validation.

A.F.: visualization, validation. 


\section{REFERENCES}

1. Chuangsuwanich A, Warnnissorn M, Lohsiriwat V. Siliconoma of the breasts. Gland Surg. 2013;2(1):46-9. https://doi. org/10.3978/j.issn.2227-684X.2013.02.05

2. Brown SL, Silverman BG, Berg WA. Rupture of silicone-gel breast implants: causes, sequelae, and diagnosis. Lancet. 1997;350(9090):1531-7. https://doi.org/10.1016/S0140 6736(97)03164-4

3. Carson B, Cox S, Ismael H. Giant siliconoma mimicking locally advanced breast cancer: a case report and review of literature. Int J Surg Case Rep. 2018;48:54-60. https://doi.org/10.1016/j. ijscr.2018.05.001

4. Lee Y, Song SE, Yoon E-S, Bae JW, Jung SP. Extensive silicone lymphadenopathy after breast implant insertion mimicking malignant lymphadenopathy. Ann Surg Treat Res. 2017;93(6):331-5. https://doi.org/10.4174/astr.2017.93.6.331

5. Oh JH, Song SY, Lew DH, Lee DW. Distant migration of multiple siliconomas in lower extremities following breast implant rupture: case report. Plast Reconstr Surg Glob Open. 2016;4(10):e1011.https://doi.org/10.1097/GOX.0000000000001011

6. Jeng C-J, Ko M-L, Wang T-H, Huang S-H. Vulvar siliconoma migrating from injected silicone breast augmentation. BJOG. 2005;112(12):1659-60. https://doi.org/10.1111/j.14710528.2005.00761.x

7. Grubstein A, Cohen M, Steinmetz A, Cohen D. Siliconomas mimicking cancer. Clin Imaging. 2011;35(3):228-31. https:// doi.org/10.1016/j.clinimag.2010.07.006 